\title{
Half-Cycle Pulse Acting on a One-Dimensional Rydberg Atom: Semiclassical Transition Amplitudes in Action and Angle Variables
}

\author{
C. D. Schwieters \\ William \& Mary \\ John B. Delos \\ William \& Mary, jbdelos@wm.edu
}

Follow this and additional works at: https://scholarworks.wm.edu/aspubs

Part of the Physics Commons

\section{Recommended Citation}

Schwieters, C. D. and Delos, John B., Half-Cycle Pulse Acting on a One-Dimensional Rydberg Atom: Semiclassical Transition Amplitudes in Action and Angle Variables (1995). Physical Review A, 51(2), 1030-1041.

https://doi.org/10.1103/PhysRevA.51.1030

This Article is brought to you for free and open access by the Arts and Sciences at W\&M ScholarWorks. It has been accepted for inclusion in Arts \& Sciences Articles by an authorized administrator of W\&M ScholarWorks. For more information, please contact scholarworks@wm.edu. 


\title{
Half-cycle pulse acting on a one-dimensional Rydberg atom: Semiclassical transition amplitudes in action and angle variables
}

\author{
C. D. Schwieters and J. B. Delos \\ Physics Department, The College of William and Mary, Williamsburg, Virginia 23187; \\ Joint Institute for Laboratory Astrophysics, University of Colorado, Boulder, Colorado 80309; \\ and National Institute of Standards and Technology, Boulder, Colorado 80309
}

(Received 11 August 1994)

\begin{abstract}
In this paper we derive the expression for the transition coefficient used in the preceding paper [C. D. Schwieters and J. B. Delos, Phys. Rev. A 51, 1023 (1995)] for principal-quantum-number transitions in one-dimensional hydrogen caused by half-cycle pulses. We briefly review the methods of Miller [Adv. Chem. Phys. 25, 69 (1974)] and Marcus [Chem. Phys. Lett. 7, 525 (1970); J. Chem. Phys. 54, 3965 (1971)] and then derive the result using the methods of Maslov and Fedoriuk [Semi-Classical Approximation in Quantum Mechanics (Reidel, Dordrecht, 1981)]. Also, we examine the approximate reduction of hydrogen from three to one dimension and we find a hitherto unknown correction due to the residual motion of one of the ignored degrees of freedom. We discuss the regime of validity of this one-dimensional approximation.
\end{abstract}

PACS number(s): 03.65.Sq, 34.60.+z, 42.50.Vk, 34.50.Pi

\section{INTRODUCTION}

The history of action and angle variables in quantum mechanics goes back to the origins of the theory in the $1920 \mathrm{~s}[1,2]$. While action and angle variables are frequently used in classical mechanical analysis of separable and approximately separable systems, their usefulness in quantum mechanics seems more problematical [3]. In the early 1970s Miller [4] developed a theory for the use of canonical transformations to general variables $[(p, q) \rightarrow(P(p, q), Q(p, q))]$ in semiclassical mechanics. Included in this set of transformations is the transformation to action and angle variables, $(I, \theta)$. Miller applied his methods to obtain a "classical $S$-matrix" for use in molecular scattering calculations. Substantially the same result was separately derived by Marcus [5] using a WKB approximation for the Schrödinger equation in the angle variable. While the pitfalls of action and angle variables in quantum mechanics were overlooked and complex phase factors were treated cavalierly, particularly simple and useful formulas for semiclassical transition probabilities were found when expressed in these variables. Around the same time, Maslov and Fedoriuk [6] published a treatise which rigorously developed a theory for semiclassical mechanics, including careful consideration of quantum phase. They did not, however, solve all the problems associated with quantum transformations to action-angle variables.

In the preceding paper [7], we quoted the MillerMarcus result for the transition amplitude expressed in action and angle variables for hydrogen interacting with a half-cycle pulse. In the present paper, we apply the method of Maslov and Fedoriuk to derive the transition probability in $(p, q)$ coordinates and then we make a change of variables to action and angle variables to show that the formula is correct (in this case). Thus we carry on along lines of earlier work [8] in which an in- dividual quantum result is expressed in action and angle variables for ease of qualitative understanding and ease of computation; and we hope to motivate the development of a more general theory which would further elucidate the seemingly deep role of action and angle variables in quantum mechanics. In Sec. II A we review the classical formula for transition probability, and in Sec. II B we outline the Miller-Marcus formulas for transition amplitudes. In Sec. II C, we write the rigorous Maslov result and then transform to action and angle variables to recover the formulas with correct phases.

In Sec. III we examine the one-dimensional model of the hydrogen atom. This model has been used in the past $[9,10]$ to successfully interpret experiments, but to our knowledge, a careful reduction from three to one degree of freedom of hydrogen has not appeared in the literature. In Sec. III A we explicitly perform this reduction and find a small correction which does not appear in previous work. We discuss the details of the simulations shown in [7] in Sec. III B.

\section{SEMICLASSICAL TRANSITION PROBABILITIES}

We consider a system with one degree of freedom governed by a Hamiltonian which is time dependent for $t_{0}<t<t_{p}$ and time independent otherwise. Though it is not essential, we presume that the Hamiltonian for $t \leq t_{0}$ is the same as that for $t \geq t_{p}$. When we need to be more specific, we will take the Hamiltonian to have the form

$$
\begin{aligned}
H(p, q) & =H_{0}(p, q)+V_{I}(q, t) \\
H_{0}(p, q) & =\frac{1}{2 m} p^{2}+V(q),
\end{aligned}
$$


where $V_{I}(q, t)=0$ except when $t_{0} \leq t \leq t_{p}$. We call $V_{I}(q, t)$ the pulse and we call $H_{0}(p, q)$ the initial and final Hamiltonian. We wish to derive semiclassical formulas that use classical mechanics to construct approximate transition amplitudes.

\section{A. Classical mechanics}

Starting at $t_{0}$, each point in phase space $\left(p_{0}, q_{0}\right)$ evolves to a new point $\left(p_{f}, q_{f}\right)$ at time $t_{f} \geq t_{p}$. Normally, one uses an extended phase space $\left(p, q, p_{t}, t\right)$ with the effective Hamiltonian

$$
\mathcal{H}\left(p, q, p_{t}, t\right)=H(p, q, t)+p_{t}
$$

and an auxiliary variable $s$ to replace time, so that

$$
\begin{aligned}
\frac{d t}{d s} & =\frac{\partial \mathcal{H}}{\partial p_{t}}=1 \\
\frac{d p_{t}}{d s} & =-\frac{\partial \mathcal{H}}{\partial t}=-\frac{\partial H}{\partial t} \\
\frac{d q}{d s} & =\frac{\partial \mathcal{H}}{\partial p}=\frac{\partial H}{\partial p} \\
\frac{d p}{d s} & =-\frac{\partial \mathcal{H}}{\partial q}=-\frac{\partial H}{\partial q}
\end{aligned}
$$

As $t$ goes from $t_{0}$ to $t_{f}$, any phase space point evolves on a smooth path $(p(t), q(t))$ from $\left(p_{0}, q_{0}\right)$ to $\left(p_{f}, q_{f}\right)$, and the relationship between $\left(p_{0}, q_{0}\right)$ and $\left(p_{f}, q_{f}\right)$ is a canonical transformation. It is usual practice to take the initial value of $p_{t}$ to be

$$
p_{t}\left(t_{0}\right)=-H\left(p_{0}, q_{0}, t_{0}\right)
$$

and then $p_{t}(t)$ remains always (minus) the energy of the evolving phase point $p_{t}(t)=-H(p(t), q(t), t)$ and, thus, $\mathcal{H}\left(p, q, p_{t}, t\right)=0$ for all time.

It is convenient to represent the motion in terms of the action and angle variables $(I, \theta)$. The relationship between $(p, q)$ and $(I, \theta)$ is governed by the initial and final (time-independent) Hamiltonian, $H_{0}(p, q)$. The set of points $\{p, q\}$ having $H_{0}(p, q)$ equal to some fixed value $E$ forms a closed one-dimensional loop in the twodimensional $(p, q)$ phase space. For any given value of $(p, q)$ the value of the action variable is the area enclosed by this loop divided by $2 \pi$ :

$$
I(p, q)=\frac{1}{2 \pi} \int_{H_{0}(p, q) \leq E} d p d q \equiv I(E)
$$

and the angle variable $\theta(p, q)$ is defined in such a way that for evolution under $H_{0}$ (i) $\theta$ increases linearly with time and (ii) as the phase point moves around one cycle of the loop, $\theta$ increases by $2 \pi$. The conventional integral formula for $\theta$ is obtained from the following prescription. Given $p, q$ evaluate $H_{0}(p, q)$, and then define the function $p(q ; E)$ through $H_{0}(p, q)=E$. Since

$$
d \theta / d t=2 \pi / T(E)=(d \theta / d q)(d q / d t)
$$

where $T(E)$ is the period of motion, it follows that

$$
\theta(q ; E)=\frac{2 \pi}{T(E)} \int_{q>}^{q}\left[\frac{\partial H_{0}(p, q)}{\partial p}\right]_{p=p(q ; E)}^{-1} d q .
$$

We take $q_{>}$to be the right-hand turning point, the maximum possible value of $q$ given $E$. Furthermore, it is convenient to introduce an angle variable which is independent of time by the relation

$$
\Theta(q ; E, t)=\theta(q ; E)-\omega(E) t
$$

where $\omega(E)=2 \pi / T(E)$ is the orbital (angular) frequency. The two-dimensional phase space described by $(p, q)$ may be alternatively represented by either $(I, \theta)$ or $(I, \Theta)$ through canonical transformations.

The classical analogue of an initial quantum state is the ensemble of points $\{p, q\}$ having $H_{0}(p, q)=E_{0}$, such that $I\left(E_{0}\right)=\left(n_{0}+1 / 2\right) \hbar$. This ensemble of points is called the initial Lagrangian manifold, and each point can be labeled by the value of its angle variable at $t=t_{0}$, $\Theta\left(t_{0}\right)=\Theta_{0}$. We say that the density of this ensemble is constant, which means that the probability of finding the particle within $d \Theta$ of angle $\Theta$ is independent of $\Theta$. We consider the evolution of this ensemble of points, i.e., the time development of the Lagrangian manifold under the influence of the time-dependent pulse.

When the pulse is turned on, $p$ and $q$ evolve according to the equations of motion; the Lagrangian manifold becomes a two-dimensional surface in the fourdimensional $\left(p, q, p_{t}, t\right)$ extended phase space, and locations on this surface can be specified by two variables, $\left(\Theta_{0}, t\right)$. At the time $t=t_{f}$ after the pulse has turned off, the smooth loop has evolved into a complicated loop $\left(p\left(\Theta_{0}, t_{f}\right), q\left(\Theta_{0}, t_{f}\right)\right)$ which may have many twists and folds, but which cannot intersect itself, and which encloses the same area as the original loop. For each $\Theta_{0}$, the value of $p_{t}\left(\Theta_{0}, t_{f}\right) \equiv-E\left(\Theta_{0}, t_{f}\right) \equiv-E_{f}\left(\Theta_{0}\right)$ is obtained by integration of the equations of motion [Eq. $(3 \mathrm{~b})]$, and the final action $I\left(\Theta_{0}, t_{f}\right) \equiv I_{f}\left(\Theta_{0}\right)$ is obtained from Eq. (5) with $E$ replaced by $E_{f}\left(\Theta_{0}\right)$. The classical probability for finding the particle within an interval of width $\hbar$, around one of the quantized values of action, $I_{n}=(n+1 / 2) \hbar$, is then proportional to the range of initial angle $\Delta \Theta_{0}$ that lands within that interval,

$$
\begin{aligned}
P\left(I_{n}\right) & =\frac{\Delta \Theta_{0}}{2 \pi} \\
& =\frac{1}{2 \pi}\left|\frac{d \Theta_{0}}{d I_{f}}\right| d I_{f} \\
& =\frac{\hbar}{2 \pi}\left|\frac{d I_{f}\left(\Theta_{0}\right)}{d \Theta_{0}}\right|^{-1} .
\end{aligned}
$$

\section{B. Transformation matrix elements and the semiclassical transition amplitude}

We now use Miller's method [4] to write down semiclassical formulas for quantum transition amplitudes. 
Suppose in classical mechanics we have a canonical transformation between "old" variables $(P, Q)$ and "new" variables $(p, q)$. Let $|p\rangle,|q\rangle,|P\rangle,|Q\rangle$ be eigenvectors of the corresponding quantum mechanical operators. Then it was proposed that there would be a relationship between quantum transformation matrix elements and the classical generators of the canonical transformation. For example,

$$
\begin{aligned}
\langle p \mid P\rangle & =\left(-\frac{\partial^{2} F_{4}(p, P) / \partial p \partial P}{2 \pi i \hbar}\right)^{1 / 2} \exp \left(\frac{i}{\hbar} F_{4}(p, P)\right) \\
\langle q \mid P\rangle & =\left(\frac{\partial^{2} F_{2}(q, P) / \partial q \partial P}{2 \pi i \hbar}\right)^{1 / 2} \exp \left(\frac{i}{\hbar} F_{2}(q, P)\right)
\end{aligned}
$$

and

$$
\langle p \mid q\rangle=(-2 \pi i \hbar)^{-1 / 2} \exp \left(\frac{-i p q}{\hbar}\right) .
$$

It was claimed that these transition elements would form a closed "algebra" when integrals were performed using the stationary-phase approximation, i.e.,

$$
\langle p \mid P\rangle=\int\langle p \mid q\rangle\langle q \mid P\rangle d q .
$$

There are four problems with these formulas: (1) The variables are not specifically defined (the formula is supposed to be true for general canonical transformations). (2) If $q$ is defined to be a classical angle variable then the corresponding quantum kets $|q\rangle$ do not, in general, exist. (3) The overall phase (due to the square root of $i$ ) is not defined. (4) If we attempt to define that phase by any formula such as

$$
(-i a)^{1 / 2}=|a|^{1 / 2} e^{-i(\pi / 4) \operatorname{sgn} a}
$$

then the result is not, in general, correct.

Otherwise, the formulas are correct. Moreover, they give a remarkably simple prescription for transition amplitudes.
Under the classical Hamiltonian let orbits evolve from initial action and angles $I_{0}, \Theta_{0}$ to final actions and angles $I_{f}, \Theta_{f}$. The relationship between initial and final phase points is a canonical transformation, and typically there exists a generator $F_{4}\left(I_{f}, I_{0}\right)$ such that

$$
\begin{aligned}
\Theta_{f} & =-\frac{\partial F_{4}\left(I_{f}, I_{0}\right)}{\partial I_{f}}, \\
\Theta_{0} & =\frac{\partial F_{4}\left(I_{f}, I_{0}\right)}{\partial I_{0}} .
\end{aligned}
$$

This generator is an integral of a Legendre transform of the Lagrangian

$$
F_{4}\left(I_{f}, I_{0}\right)=\int_{t_{0}}^{t_{f}}\left(-\Theta(t) \frac{d I(t)}{d t}-H(I(t), \Theta(t))\right) d t
$$

along the classical trajectory which begins at $I_{0}, \Theta_{0}$ and ends at $I_{f}, \Theta_{f}$.

The corresponding quantum evolution begins from an initial ket which might conventionally be labeled $\left|n_{0}\right\rangle$, but which we label by $\left|\left(n_{0}+1 / 2\right) \hbar\right\rangle \equiv\left|I_{0}\right\rangle$, the initial quantized value of action. This ket is normalized "on the action scale," such that

$$
\left\langle I_{0} \mid I_{0}\right\rangle=\hbar^{-1} .
$$

As stated before, $\left|I_{0}\right\rangle$ corresponds to an ensemble of trajectories having initial action $I_{0}$ and any initial value of angle $\Theta_{0}$. Under the quantum Hamiltonian this ket evolves to a new ket which we call $\left|I_{0}(t)\right\rangle$. Then the probability of finding the system in the final state $\left|I_{f}=I_{n}\right\rangle$ is

$$
P\left(I_{f}\right)=\hbar^{2}\left|\left\langle I_{f} \mid I_{0}(t)\right\rangle\right|^{2} .
$$

The factor $\hbar^{2}$ comes from the normalization [Eq. (15)].

The semiclassical formula for this transition probability comes from Eq. (10a) with the interpretations

$$
\begin{aligned}
\langle p| \rightarrow\left\langle I_{f}\right|, \quad p & \rightarrow I_{f}, \\
|P\rangle & \rightarrow\left|I_{0}(t)\right\rangle, P \rightarrow I_{0} .
\end{aligned}
$$

We obtain directly

$$
\begin{aligned}
\left\langle I_{f} \mid I_{0}(t)\right\rangle & =\left(-\frac{\partial^{2} F_{4}\left(I_{f}, I_{0}\right) / \partial I_{f} \partial I_{0}}{2 \pi i \hbar}\right)^{1 / 2} \exp \left[i F_{4}\left(I_{f}, I_{0}\right) / \hbar\right] \\
& =\left(-2 \pi i \hbar \frac{\partial I_{f}}{\partial \Theta_{0}}\right)^{-1 / 2} \exp \left[i / \hbar \int_{t_{0}}^{t_{f}}\left(-\Theta(t) \frac{d I}{d t}-H(t)\right) d t\right] .
\end{aligned}
$$

These quantities are all defined for continuous values of $I_{f}$; they are to be evaluated at the discrete values $I_{f}=I_{n}$. If there is more than one value of $\Theta_{0}$ having a trajectory connecting $I_{0}$ to $I_{f}=I_{n}$, then $\left\langle I_{f} \mid I_{0}\left(t_{f}\right)\right\rangle$ is a sum of such terms. This is the semiclassical formula for the transition amplitude, and despite all the objections listed above, it is a good formula. Noting the normalization in Eq. (16), the magnitude of Eq. (18b) goes to the correct classical limit [Eq. (9)], and even the phases are correct when we use the convention from Eq. (12).

Near any local extremum of the function $I_{f}\left(\Theta_{0}\right)$, $d I_{f} / d \Theta_{0}$ vanishes and the formula fails. In such regions we write

$$
\left\langle I_{f} \mid I_{0}(t)\right\rangle=\int\left\langle I_{f} \mid \Theta_{f}\right\rangle\left\langle\Theta_{f} \mid I_{0}(t)\right\rangle d \Theta_{f}
$$


Ignoring questions about the existence of the kets $\left|\Theta_{f}\right\rangle$, we write from Eq. (10c)

$$
\left\langle I_{f} \mid \Theta_{f}\right\rangle=(-2 \pi i \hbar)^{-1 / 2} \exp \left[-i I_{f} \Theta_{f} / \hbar\right]
$$

and use Eq. (10b) to obtain

$$
\begin{aligned}
\left\langle\Theta_{f} \mid I_{0}(t)\right\rangle= & {\left[\frac{\partial^{2} F_{2}\left(\Theta_{f}, I_{0}\right) / \partial \Theta_{f} \partial I_{0}}{2 \pi i \hbar}\right]^{1 / 2} } \\
& \times \exp \left[i F_{2}\left(\Theta_{f}, I_{0}\right) / \hbar\right] .
\end{aligned}
$$

In this case

$$
F_{2}\left(\Theta_{f}, I_{0}\right)=\int_{t_{0}}^{t_{f}}\left(I(t) \frac{d \Theta(t)}{d t}-H(t)\right) d t+I_{0} \Theta_{0}
$$

and

$$
\frac{\partial^{2} F_{2}}{\partial \Theta_{f} \partial I_{0}}=\left(\frac{\partial \Theta_{0}}{\partial \Theta_{f}}\right)
$$

so that Eq. (19) gives an integral representation of the transition amplitude

$$
\begin{aligned}
\left\langle I_{f} \mid I_{0}(t)\right\rangle= & (-2 \pi i \hbar)^{-1 / 2} \int d \Theta_{f}\left(\frac{\partial \Theta_{0} / \partial \Theta_{f}}{2 \pi i \hbar}\right)^{1 / 2} \\
& \times \exp \left\{i\left[F_{2}\left(\Theta_{f}, I_{0}\right)-I_{f} \Theta_{f}\right] / \hbar\right\}
\end{aligned}
$$

If we change the variable of integration from $\Theta_{f}$ to $\Theta_{0}$ we obtain

$$
\begin{aligned}
\left\langle I_{f} \mid I_{0}(t)\right\rangle= & (-2 \pi i \hbar)^{-1 / 2} \int d \Theta_{0}\left(\frac{\partial \Theta_{f} / \partial \Theta_{0}}{2 \pi i \hbar}\right)^{1 / 2} \\
& \times \exp \left\{i\left[F_{2}\left(\Theta_{f}, I_{0}\right)-I_{f} \Theta_{f}\right] / \hbar\right\}
\end{aligned}
$$

Except for Maslov phases, these expressions can be reduced to the primitive semiclassical approximation [Eq. $(18 \mathrm{~b})$ ] by using the stationary-phase approximation. We explicitly evaluate the integral in Eq. (23) using this approximation to obtain

$$
\begin{aligned}
\left\langle I_{f} \mid I_{0}(t)\right\rangle \sim & \sqrt{2 \pi \hbar}(2 \pi i \hbar)^{-1 / 2}\left(\frac{\partial \Theta_{0} / \partial \Theta_{f}}{2 \pi i \hbar}\right)^{1 / 2}\left|\frac{\partial^{2} F_{2}\left(\Theta_{f}, I_{0}\right)}{\partial \Theta_{f}^{2}}\right|^{-1 / 2} e^{i(\pi / 4) \operatorname{sgn}\left(\partial^{2} F_{2} / \partial \Theta_{f}^{2}\right)} \\
& \times \exp \left\{i\left[F_{2}\left(\Theta_{f}, I_{0}\right)-I_{f} \Theta_{f}\right] / \hbar\right\} \\
= & (2 \pi \hbar)^{-1 / 2}\left|\frac{\partial I_{f}}{\partial \Theta_{0}}\right|^{-1 / 2} \exp \left\{i\left[F_{2}\left(\Theta_{f}, I_{0}\right)-I_{f} \Theta_{f}\right] / \hbar\right\} \\
& \times \exp \left(i \pi / 4-i \frac{\pi}{4} \operatorname{sgn} \frac{\partial \Theta_{f}}{\partial \Theta_{0}}+i \frac{\pi}{4} \operatorname{sgn} \frac{\partial I_{f}}{\partial \Theta_{f}}\right)
\end{aligned}
$$

where a sum over all stationary-phase points satisfying $\partial F_{2} / \partial \Theta_{f}=I_{f}\left(=I_{n}\right)$ is implied. If this formula is compared with Eq. (18b), it is seen that the amplitudes match and that the phase portion due to the classical generators corresponds through a Legendre transform. However, the "Maslov" phase portion differs. Explicitly,

$$
\frac{\pi}{4} \operatorname{sgn} \frac{\partial I_{f}}{\partial \Theta_{0}} \neq \frac{\pi}{4}\left(1-\operatorname{sgn} \frac{\partial \Theta_{f}}{\partial \Theta_{0}}+\operatorname{sgn} \frac{\partial I_{f}}{\partial \Theta_{f}}\right) .
$$

The reader who goes from Eq. (23) to Eq. (25) may be tempted to try to obtain consistent results by prescribing other phase conventions in Eqs. (10), (12), and (20); there are $2^{5}$ possibilities, so one can entertain oneself for a long time. However, there exists no consistent prescription in which the phase of the wave function is defined solely in terms of local properties of the Lagrangian manifold. Indeed, that fact was a main point of Maslov's workhe found a way of defining all of the phases such that they came out consistently in stationary-phase transformations. The correct phase is given by a Maslov index, which is not a property of the local sign of $\partial^{2} F_{4} / \partial p \partial P$; rather it is a property of a path on a Lagrangian manifold, and it depends on how the manifold is connected.

Marcus [5] follows a different route to obtain the matrix element $\left\langle I_{f} \mid I_{0}\right\rangle$. He starts from the assumption that one can write a Schrödinger equation for a wave func- tion $\psi(\Theta)$ that depends upon an angle variable, and he presumes that the quantum Hamiltonian operator is the classical Hamiltonian function $H(I, \Theta)$ with the operator replacement $I \rightarrow-i \hbar \partial / \partial \Theta+\delta \hbar$. This approach has the same problems as Miller's. The function $\psi(\Theta)$ corresponds to Miller's $\left\langle\Theta \mid I_{0}\right\rangle$, and if the kets $\{|\Theta\rangle\}$ do not exist, it is not clear that any well-defined function $\psi(\Theta)$ exists. Furthermore, even if the Hamiltonian operator is defined at all in this representation, it is not clear that the above operator replacement in the classical Hamiltonian is the correct prescription.

Finally, while it is difficult to identify the phase prescriptions used by Marcus, it is evident that he was not using Maslov's prescription (which was not generally available at the time), so the formulas cannot always hold together consistently.

The derivations given by Miller and Marcus are simple, elegant, and appealing. Furthermore, except for Maslov phases, the results are correct, at both the primitive and the uniform semiclassical levels. Clearly there is something very deep about the correspondence principle that we do not yet know.

\section{Semiclassical wave functions and the transition amplitude}

In this section, we derive Eq. (18b) starting from a different perspective. It takes more effort, but everything 
is well defined. The strategy is simple: the Schrödinger equation is a second-order differential equation in $q$ space, semiclassical wave functions can be constructed in this space, and the transition probability can be expressed as an integral over $q$,

$$
\begin{aligned}
P\left(I_{n}\right) & =\hbar^{2}\left|\left\langle I_{n} \mid I_{0}(t)\right\rangle\right|^{2} \\
& =\hbar^{2}\left|\int \psi_{I_{n}}^{*}(q) \psi_{I_{0}}(q, t) d q\right|^{2} .
\end{aligned}
$$

Using the method of Maslov and Fedoriuk [6], everything in this integral can be expressed in terms of properties of the final Lagrangian manifold, the classical curve in $(p, q)$ space at time $t_{f}$ that evolves from the initial manifold. After everything is expressed in terms of classical quantities, we can finally transform to action-angle variables; the essential properties of the manifold then are contained in the functions $I_{f}\left(\Theta_{0}\right)$ and $\Theta_{f}\left(\Theta_{0}\right)$. The variable of integration $d q$ in Eq. (27) is converted to $d \Theta_{0}$ by the relation $q=q\left(t_{f}, \Theta_{0}\right)$. All classical functions of interest on the manifold are single-valued functions of $\Theta_{0}$. We thereby obtain an integral expression for the transition coefficient. We then show that, to within the accuracy of uniform semiclassical approximations, the integral expression is the same as the Miller-Marcus formula, except that the correct Maslov phases are incorporated. If the stationary-phase approximation is used for this integral, it reduces to the primitive semiclassical approximation [Eq. (18b)].

\section{Semiclassical wave functions}

As stated earlier, the initial Lagrangian manifold is a simple loop in $(p, q)$ space and for each point $q$ there are two values of $p$ labeled $p_{k}\left(q ; E_{0}\right)$ and two associated generators

$$
S_{k}\left(q ; E_{0}\right)=\int_{q>}^{q} p_{k}\left(q^{\prime} ; E_{0}\right) d q^{\prime}
$$

each of which contributes a term to the initial wave function

$$
\begin{gathered}
\psi_{I_{0}}(q)=\sum_{k=1}^{2} A_{k}\left(q ; E_{0}\right) \exp \left[i S_{k}\left(q ; E_{0}\right) / \hbar-i \mu_{k} \pi / 2\right] \\
A_{k}(q)=\left|\hbar T\left(E_{0}\right)\left[\frac{\partial H_{0}(p, q)}{\partial p}\right]_{p=p\left(q ; E_{0}\right)}\right|^{-1 / 2} .
\end{gathered}
$$

[Whenever we speak of "two" values of $p$, "two" generators, etc., we are presuming that $H_{0}(p, q)$ has the form of Eq. (1) (i.e., it is quadratic in momentum) and that the initial Lagrangian manifold is a constant-energy curve of $H_{0}(p, q)$.] Since the angle $\Theta_{0}$ is the natural variable for the initial Lagrangian manifold, we can use Eq. (8) to relate $\Theta_{0}$ to $q$. Then each quantity in Eq. (29) can be regarded as a function of $\Theta_{0}$ :

$$
\begin{aligned}
& S_{k}\left(q ; E_{0}\right)=S_{0}\left(\Theta_{0}\right), \\
& A_{k}\left(q ; E_{0}\right)=A_{0}\left(\Theta_{0}\right) \text {, } \\
& \mu_{k} \quad=\mu_{0}\left(\Theta_{0}\right) \text {. }
\end{aligned}
$$

The corresponding final state $\psi_{I_{n}}(q)$ (which is an eigenfunction of the same $H_{0}$ ) uses all the same formulas, but with $E_{0}$ replaced by $E_{n}$. If we reexpress the quantities in terms of the angle variable, we must note that the relationship between $\Theta_{n}$ and $q$ contains $E_{n}$, so $\Theta_{0}=\Theta\left(q ; E_{0}\right)$ and $\Theta_{n}=\Theta\left(q ; E_{n}\right)$ are different functions of $q$. We write

$$
\begin{aligned}
& S_{j}\left(q ; E_{n}\right)=S_{n}\left(\Theta_{n}\right), \\
& A_{j}\left(q ; E_{n}\right)=A_{n}\left(\Theta_{n}\right) \text {, } \\
& \mu_{j} \quad=\mu_{n}\left(\Theta_{n}\right) \text {. }
\end{aligned}
$$

When the pulse is turned on, the initial wave function evolves according to the familiar semiclassical rules. For each point on the final Lagrangian manifold there is a generator $S$ which could be regarded as a function of $\Theta_{0}$ and $t_{f}$. It is equal to the generator at the corresponding initial point $S\left(\Theta_{0}, t_{0}\right)$ plus the integral of action along the classical path

$$
S\left(\Theta_{0}, t_{f}\right)=S_{0}\left(\Theta_{0}\right)+\int_{t_{0}}^{t_{f}}\left(p d q+p_{t} d t\right) .
$$

The change in the amplitude is given by

$$
A\left(\Theta_{0}, t_{f}\right)=A\left(\Theta_{0}, t_{0}\right)\left|\frac{J\left(\Theta_{0}, t_{0}\right)}{J\left(\Theta_{0}, t_{f}\right)}\right|^{1 / 2},
$$

where

$$
J\left(\Theta_{0}, t\right)=\frac{\partial q\left(\Theta_{0}, t\right)}{\partial \Theta_{0}}
$$

and $q\left(\Theta_{0}, t\right)$ is the location of the particle on the path that begins at $\left(\Theta_{0}, t_{0}\right)$.

The Maslov index is also incremented appropriately along the classical path. We can also find relative values of this index at various points on the manifold for any fixed time. We examine at fixed $t$ the Lagrangian curve $\left(p\left(t, \Theta_{0}\right), q\left(t, \Theta_{0}\right)\right)$. The Maslov index changes at each point that the curve rises vertically above the $q$ axis $\left[\partial q\left(t, \Theta_{0}\right) / \partial \Theta_{0}=0\right]$. If at any such point we move along the curve in such a direction that the curve bends to the right, the Maslov index increases by 1 ; if the curve bends to the left, it decreases by 1 (Fig. 1 ).

This function $q\left(\Theta_{0}, t_{f}\right)$ connects the amplitude $A\left(\Theta_{0}, t_{f}\right)$, action $S\left(\Theta_{0}, t_{f}\right)$, and Maslov index defined on the manifold to a location in space. The evolved wave function is a sum of terms, one from each section of the final Lagrangian curve that has a projection onto that point,

$\psi_{I_{0}}(q, t)=\sum_{k} A_{k}\left(q, t_{f}\right) \exp \left[i S_{k}\left(q, t_{f}\right) / \hbar-i \mu_{k} \pi / 2\right]$,

where $A_{k}\left(q, t_{f}\right)=A_{k}\left(q\left(\Theta_{0}, t_{f}\right), t_{f}\right)$ and similarly for $S_{k}$ and $\mu_{k}$. If the evolved manifold is very convoluted, there may be several terms in the wave function contributing at each point $q$.

Then the transition probability [Eq. (27)] is the absolute square of the sum of interfering terms: 


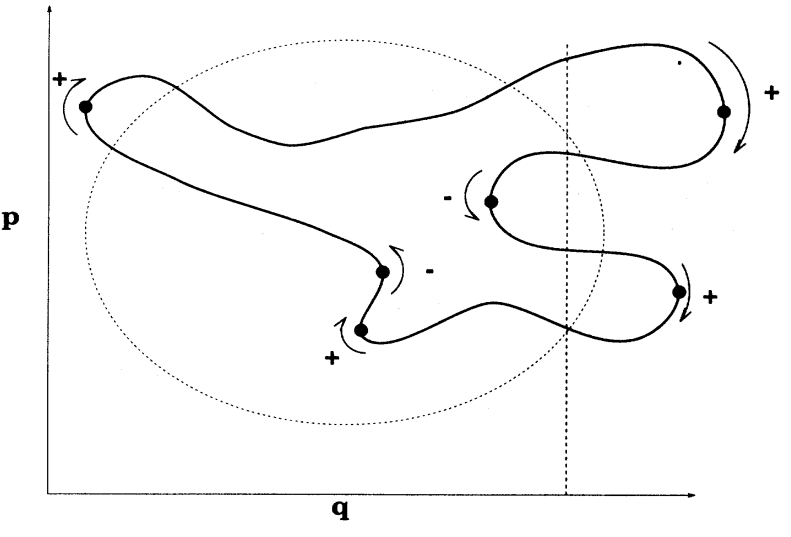

FIG. 1. How the Maslov index is incremented on a Lagrangian manifold. We choose to traverse the curve in the clockwise direction and the Maslov index changes as indicated when $(\partial p / \partial q \rightarrow \infty)$. The dashed loop represents the final-state manifold while the solid loop represents the time-evolved manifold.

$$
\begin{aligned}
P\left(I_{n}\right) & =\left|a_{n}\right|^{2}, \\
a_{n} & =\int d q\left[\sum_{j k} f_{j k}(q) \exp \left(i g_{j k}(q) / \hbar-i \mu_{j k} \frac{\pi}{2}\right)\right], \\
f_{j k} & =\hbar A_{j}\left(q ; E_{n}\right) A_{k}\left(q ; t_{f}\right), \\
g_{j k} & =S_{k}\left(q ; t_{f}\right)-S_{j}\left(q ; E_{n}\right), \\
\mu_{j k} & =\mu_{k}-\mu_{j} .
\end{aligned}
$$

We will show that a phase-corrected Miller-Marcus formula is "semiclassically equivalent" to this formula.

\section{Semiclassical equivalence}

Given an integral of the form

$$
J=\int F(q) \exp [i G(q) / \hbar] d q,
$$

semiclassical theories use an expansion in powers of $\hbar$ in which the first few terms in the expansion are integrals of some standard type. A general framework for accomplishing this was developed by Chester, Friedman, and Ursell $[11,12]$ and applied to atomic and molecular problems by Connor [13]. Their method involves examination of the stationary-phase points of the $G(q)$ function, at which $d G(q) / d q=0$. For example, in the case considered in Figs. 5 and 6 in the preceding paper, there are three stationary-phase points close together so the appropriate mapping $q \rightarrow x$ is quartic,

$$
G(q(x))=\frac{1}{4} x^{4}-\xi x^{2}-\eta x+A,
$$

where $\xi, \eta$, and $A$ are found by forcing $G(q)$ and $G(q(x))$ to match at the stationary-phase points. To within errors of order $\hbar^{5 / 4}$ the integral is given approximately by a linear combination of a certain canonical integral and its derivatives

$J \approx[\alpha U(\xi, \eta)+\beta \partial U(\xi, \eta) / \partial \eta+\gamma \partial U(\xi, \eta) / \partial \xi] e^{i A}$,

where the canonical integral is the Pearcey function [14]

$$
U(\xi, \eta)=\int_{-\infty}^{\infty} \exp \left[i\left(\frac{1}{4} x^{4}-\xi x^{2}-\eta x\right)\right] d x .
$$

The parameters $(\xi, \eta)$ in the mapping from $q$ to $x$ are determined by differences in $G(q)$ function evaluated at the stationary-phase points, $G\left(q_{k}\right)-G\left(q_{j}\right)$. The coefficients $(\alpha, \beta, \gamma)$ are determined by the product $F\left(q_{k}\right)\left|d^{2} G\left(q_{k}\right) / d q^{2}\right|^{-1 / 2}$ evaluated at the stationaryphase points, in addition to $\xi$ and $\eta$.

Therefore, we may say that two different integrals

$$
\begin{aligned}
& J_{1}=\int F_{1}(y) \exp \left[i G_{1}(y) / \hbar\right] d y, \\
& J_{2}=\int F_{2}(z) \exp \left[i G_{2}(z) / \hbar\right] d z
\end{aligned}
$$

are "semiclassically equivalent" if they have the same reduction to canonical integrals, i.e., if (a) they have the same number of stationary-phase points, (b) differences in the $G(q)$ functions evaluated at stationaryphase points are the same, and (c) the quantity $F(q)\left|d^{2} G(q) / d q^{2}\right|^{-1 / 2}$ evaluated at the stationary-phase points is the same. Then the integrals yield the same result to within $\hbar^{1+1 / M}$, where $M-1$ is the number of stationary-phase points considered [12].

\section{Corrected Miller-Marcus formula}

We stated earlier that the Miller-Marcus formula is correct provided that the phase is adjusted by Maslov indices. The phase-corrected formula is

$$
\begin{aligned}
a_{n}= & \frac{1}{2 \pi} \int d \Theta_{0}\left|\frac{\partial \Theta_{f}}{\partial \Theta_{0}}\right|_{I_{0}}^{1 / 2} \\
& \times \exp \left\{\frac{i}{\hbar}\left[F_{2}\left(\Theta_{f}, I_{0}\right)-I_{n} \Theta_{f}\left(I_{0}, \Theta_{0}\right)\right]\right. \\
& \left.-i \hat{\mu}\left[q_{f}\left(\Theta_{0}\right)\right] \pi / 2\right\} \\
= & \int \hat{f}\left(\Theta_{0}\right) \exp \left\{\frac{i}{\hbar} \hat{g}\left(\Theta_{0}\right)-i \hat{\mu}\left[q\left(\Theta_{0}\right)\right] \pi / 2\right\} d \Theta_{0}
\end{aligned}
$$

We propose that this formula is semiclassically equivalent to Eq. (37).

(a) Same number of stationary-phase points. The stationary-phase points for the Miller-Marcus formula occur at

$$
\begin{aligned}
\frac{d \hat{g}\left(\Theta_{0}\right)}{d \Theta_{0}} & =\left(\frac{\partial F_{2}}{\partial \Theta_{f}}-I_{n}\right) \frac{\partial \Theta_{f}\left(I_{0}, \Theta_{0}\right)}{\partial \Theta_{0}} \\
& =\left[I_{f}\left(I_{0}, \Theta_{0}\right)-I_{n}\right] \frac{\partial \Theta_{f}\left(I_{0}, \Theta_{0}\right)}{\partial \Theta_{0}}=0 .
\end{aligned}
$$

Stationary-phase points at which $\partial \Theta_{f} / \partial \Theta_{0}=0$ do not contribute because of the preexponential factor in Eq. (43). The other points, where $I_{f}\left(I_{0}, \Theta_{0}\right)=I_{n}$, are points 
where the evolved Lagrangian manifold intersects the manifold representing the final state.

For each such point there is a term $(j, k)$ in Eq. (37) containing a corresponding stationary-phase point:

$$
\begin{aligned}
\frac{d g_{j k}(q)}{d q} & =\frac{\partial S_{k}\left(q ; t_{f}\right)}{\partial q}-\frac{\partial S_{j}\left(q ; E_{n}\right)}{\partial q} \\
& =p_{k}\left(q ; t_{f}\right)-p_{j}\left(q ; E_{n}\right)=0 .
\end{aligned}
$$

Since the first term represents the momentum at some point on the evolved manifold and the second represents the momentum on the manifold for the final state $\left\langle I_{n}\right|$, this term again vanishes at points where these manifolds intersect.

(b) Same phase differences. In Eq. (37), the phase difference between successive manifold intersections is equal to the area enclosed between the two manifolds in $(p, q)$ space. Such areas are Poincaré/Cartan invariants and as such are conserved under canonical transformations [15]. In Eq. (43) the phase difference is the corresponding area in $\left(I_{f}, \Theta_{f}\right)$ space. Since $(p, q) \leftrightarrow\left(I_{f}, \Theta_{f}\right)$ are related by a canonical transformation, these areas are equal.

We make the Maslov phases equal by asserting that the proper quantity $\hat{\mu}\left(\Theta_{0}\right)$ to use in Eq. (43) is the one that makes the total phase at each stationary-phase point in Eq. (43) equal to the total phase at the corresponding point in Eq. (37).

(c) Same amplitudes. Now let us examine the quantities $f_{j k}(q)\left|d^{2} g_{j k}(q) / d q^{2}\right|^{-1 / 2}$ and $\hat{f}\left(\Theta_{0}\right)\left|d^{2} \hat{g}\left(\Theta_{0}\right) / d \Theta_{0}^{2}\right|^{-1 / 2}$ evaluated at the stationaryphase points. Substituting the expressions for wave function amplitude into (37c), we obtain

$$
\begin{aligned}
f_{j k}(q)= & \hbar A_{j}\left(q ; E_{n}\right) A_{k}\left(q ; t_{f}\right) \\
= & \frac{\sqrt{\omega_{0} \omega_{n}}}{2 \pi}\left|\left(\frac{\partial H_{0}}{\partial p}\right)_{p=p\left(q ; E_{n}\right)}\right|^{-1 / 2} \\
& \times\left|\frac{\partial q\left(\Theta_{0}, t_{0}\right)}{\partial \Theta_{0}} / \frac{\partial q\left(\Theta_{0}, t_{f}\right)}{\partial \Theta_{0}}\right|^{1 / 2} \\
& \times\left|\left(\frac{\partial H_{0}}{\partial p}\right)_{p=p\left(q_{0} ; E_{0}\right)}^{-1 / 2}\right|^{-}
\end{aligned}
$$

where subscript 0 indicates that the quantity is evaluated at $t=0$ while the subscript $f$ denotes evaluation at the final time. Now we note that

$$
\left(\frac{\partial q_{0}}{\partial \Theta_{0}}\right)_{I_{0}}=\frac{d q_{0}}{d t_{1}} / \frac{d \Theta_{0}}{d t_{1}}=\frac{1}{\omega\left(E_{0}\right)}\left(\frac{\partial H_{0}}{\partial p}\right)_{p=p\left(q_{0} ; E_{0}\right)}
$$

where $t_{1}$ is a timelike parameter on the initial manifold. Therefore,

$$
\begin{aligned}
f_{j k}(q)= & \frac{\sqrt{\omega_{n}}}{2 \pi}\left|\left(\frac{\partial H_{0}}{\partial p}\right)_{p=p\left(q ; E_{n}\right)}\right|^{-1 / 2} \\
& \times\left|\frac{\partial q\left(\Theta_{0}, t_{f}\right)}{\partial \Theta_{0}}\right|^{-1 / 2} .
\end{aligned}
$$

The second derivative of the phase term gives

$$
\frac{\partial^{2}}{\partial q^{2}} g_{j k}=\left(\frac{\partial p_{f}}{\partial q}-\frac{\partial p_{n}}{\partial q}\right)
$$

Recall that these partial derivatives are taken at constant $I_{0}$. Let us define a function on the final time manifold

$$
E_{f}(q) \equiv H\left(p_{f}(q), q\right)
$$

Taking the derivative with respect to $q$ and solving for $\left(\partial p_{f} / \partial q\right)_{I_{0}}$, we obtain

$$
\left(\frac{\partial p_{f}}{\partial q}\right)_{I_{0}}=\left(\frac{\partial H}{\partial p}\right)_{q}^{-1}\left[\left(\frac{\partial E_{f}}{\partial q}\right)_{I_{0}}-\left(\frac{\partial H}{\partial q}\right)_{p}\right] .
$$

Introducing a timelike parameter $t_{2}$ on the final-state manifold, we express $\left(\partial p_{n} / \partial q\right)_{I_{n}}$ as

$$
\left(\frac{\partial p_{n}}{\partial q}\right)_{I_{n}}=\frac{d p_{n}}{d t_{2}}\left(\frac{d q}{d t_{2}}\right)^{-1}=-\frac{\partial H_{0}}{\partial q}\left(\frac{\partial H_{0}}{\partial p}\right)^{-1} .
$$

Substituting these expressions into Eq. (49), we obtain

$$
\frac{\partial^{2}}{\partial q^{2}} g_{j k}=\left(\frac{\partial H}{\partial p}\right)^{-1}\left(\frac{\partial E_{f}}{\partial q}\right)_{I_{0}} .
$$

Combining Eq. (53) with (48) one then finds

$$
\begin{aligned}
f_{j k}(q)\left|\frac{d^{2} g_{j k}(q)}{d q^{2}}\right|^{-1 / 2} & =\frac{\sqrt{\omega_{n}}}{2 \pi}\left|\frac{\partial q_{f}}{\partial \Theta_{0}}\right|^{-1 / 2}\left|\frac{\partial E_{f}}{\partial q}\right|^{-1 / 2} \\
& =\frac{1}{2 \pi}\left|\frac{\partial I_{f}}{\partial \Theta_{0}}\right|^{-1 / 2}
\end{aligned}
$$

with the last simplification made possible by noting that

$$
\frac{\partial E_{f}}{\partial \Theta_{0}}=\frac{\partial E_{f}}{\partial I_{f}} \frac{\partial I_{f}}{\partial \Theta_{0}}
$$

and that $\partial E_{f} / \partial I_{f}=1 / \omega_{n}$ at the manifold crossings.

The corresponding quantity from the Miller-Marcus formula is

$$
\begin{aligned}
\hat{f}\left(\Theta_{0}\right)\left|\frac{\partial^{2} \hat{g}\left(\Theta_{0}\right)}{\partial \Theta_{0}^{2}}\right|^{-1 / 2} & =\frac{1}{2 \pi}\left|\frac{\partial \Theta_{f}}{\partial \Theta_{0}}\right|^{1 / 2}\left|\frac{\partial I_{f}}{\partial \Theta_{0}} \frac{\partial \Theta_{f}}{\partial \Theta_{0}}\right|^{-1 / 2} \\
& =\frac{1}{2 \pi}\left|\frac{\partial I_{f}}{\partial \Theta_{0}}\right|^{-1 / 2}
\end{aligned}
$$

Thus the phase-corrected Miller-Marcus formula for transition coefficient [Eq. (43)] is semiclassically equivalent to the result obtained by the method of Maslov [Eq. (37)]. Our analysis presumed that the configuration space form of the semiclassical approximation is valid. This assumption fails near turning points, but there the momentumspace semiclassical approximation can be used. The expression for the transition coefficient obtained by taking the overlap of such wave functions may also be transformed to action and angle variables, as in the above analysis and, in fact, yields the same result as quoted above in Eq. (43). 
Note that the action-angle representation of the transition coefficient depends only upon $I_{f}\left(\Theta_{0}, I_{0}\right)$ and $\Theta_{f}\left(\Theta_{0}, I_{0}\right)$ and that both of these are single-valued functions of $\Theta_{0}$. Because $I_{f}$ and $\Theta_{f}$ are independent of time after the pulse, $t>t_{p}$, it is evident that Eq. (43) is independent of final time, as expected. This fact is not immediately evident from the expression for $a_{n}$ in terms of $q$ [Eq. (37)] because it involves quantities defined on $\left(p_{f}, q_{f}\right)$ manifolds which continue to evolve after the pulse has ended.

Once again, we note that the topological phase denoted by $\hat{\mu}\left[q\left(\Theta_{0}\right)\right]$ has not been expressed in action and angle variables: it must be externally determined from the configuration or momentum-space wave functions. This term reflects the number of singularities encountered on a directed path on a manifold [6] and the difference between these terms evaluated at stationary-phase points represents the number of such singularities on an arbitrary path between stationary-phase points on a manifold in $(p, q)$, as discussed in the preceding section. Under canonical transformations the topology of the relevant manifolds changes radically, and we know of no consistent treatment of topological phase under general canonical transformations. However, the need to refer back to the $(p, q)$ manifolds is eliminated once the stationaryphase approximation has been made, as is shown below.

If the stationary-phase points are sufficiently far from each other, the stationary-phase approximation may be used to evaluate both the Miller-Marcus and the Maslov expressions for the transition coefficients [Eq. (43) and Eq. (37)]. A careful analysis of the phase factors reveals that the correct "Maslov" phase in the resulting expression is $-\frac{i \pi}{4} \operatorname{sgn}\left(\partial I_{f} / \partial \Theta_{0}\right)$ which is the complex phase intended by the square root in Eq. (18b). Thus in this example it becomes unnecessary to refer to the configuration space wave functions in the primitive semiclassical approximation.

We have thus shown that the rigorous analysis along the lines of Maslov for the transition coefficient in the preceding section is equivalent to that obtained using the methods of Miller [4] and Marcus [5] shown in Eq. (18b) in the stationary-phase approximation to within the normalization factor [Eq. (15)].

\section{A HALF-CYCLE PULSE INTERACTING WITH ONE-DIMENSIONAL HYDROGEN}

Here we apply the semiclassical theory developed in the preceding section to quasi-one-dimensional states of hydrogen in parallel static and time-dependent fields. We first analyze the Hamiltonian for the full threedimensional system and we show how it approximately reduces to one dimension. We also discuss special features of this system and how the formulas were evaluated numerically.

\section{A. Classical description \\ 1. Equations of motion}

We now examine the two-dimensional (2D) classical Hamiltonian for hydrogen in parallel static and time- varying electric fields, where the ignorable $\phi$ motion about the $z$ axis has already been removed. In cylindrical coordinates, the classical Hamiltonian is

$$
\begin{aligned}
H_{2 \mathrm{D}}= & \frac{1}{2 \mu}\left(p_{z}^{2}+p_{\rho}^{2}+l_{z}^{2} / \rho^{2}\right)-e^{2} /\left(\rho^{2}+z^{2}\right)^{1 / 2} \\
& +z e\left[\mathcal{F}_{1}+\mathcal{F}_{2}(t)\right]
\end{aligned}
$$

where $\mathcal{F}_{1}$ and $\mathcal{F}_{2}(t)$ are the weak static field and the pulse, and $l_{z}$ is the component of angular momentum about the $z$ axis, which we take to be zero in the current study.

We make the canonical transformation to semiparabolic coordinates $u$ and $v$,

$$
\begin{gathered}
u=(r+z)^{1 / 2}, v=(r-z)^{1 / 2}, \\
p_{u}=p_{\rho} v+p_{z} u, \quad p_{v}=p_{\rho} u-p_{z} v,
\end{gathered}
$$

and write the Hamiltonian in an extended phase space, as was done in Sec. II A which includes time as a coordinate by adding to the Hamiltonian the momentum conjugate to time, $p_{t}=-E(t)$. The resulting transformed Hamiltonian is

$$
\begin{aligned}
\mathcal{H}= & \frac{1}{2 \mu} \frac{p_{u}^{2}+p_{v}^{2}-4 e^{2} \mu}{u^{2}+v^{2}} \\
& +\frac{e}{2}\left(u^{2}-v^{2}\right)\left[\mathcal{F}_{1}+\mathcal{F}_{2}(t)\right]+p_{t}=0
\end{aligned}
$$

The variable $t$ (time) is now replaced by $s$ as the variable denoting parametric displacement along a trajectory. Finally, to remove the singularity along the axes $(u=0$ and $v=0$ ), we make a transformation of the time parameter $s \rightarrow \tau$, described by

$$
\frac{d s}{d \tau}=u^{2}+v^{2}
$$

and the Hamiltonian becomes

$$
\begin{aligned}
\tilde{H}=\frac{d s}{d \tau} \mathcal{H}= & \frac{1}{2 \mu}\left(p_{u}^{2}+p_{v}^{2}\right)+\frac{e}{2}\left(u^{4}-v^{4}\right)\left[\mathcal{F}_{1}+\mathcal{F}_{2}(t)\right] \\
& +\left(u^{2}+v^{2}\right) p_{t}-2 e^{2} \\
= & 0
\end{aligned}
$$

Hamilton's equations then read

$$
\begin{aligned}
\frac{d}{d \tau} u & =\frac{1}{\mu} p_{u} \\
\frac{d}{d \tau} v & =\frac{1}{\mu} p_{v} \\
\frac{d}{d \tau} p_{u} & =-2 u^{3} e\left[\mathcal{F}_{1}+\mathcal{F}_{2}(t)\right]-2 u p_{t} \\
\frac{d}{d \tau} p_{v} & =2 v^{3} e\left[\mathcal{F}_{1}+\mathcal{F}_{2}(t)\right]-2 v p_{t} \\
\frac{d}{d \tau} t & =u^{2}+v^{2} \\
\frac{d}{d \tau} p_{t} & =-\frac{e}{2}\left(u^{4}-v^{4}\right) \frac{\partial}{\partial t} \mathcal{F}_{2}(t)
\end{aligned}
$$

If $\mathcal{F}_{2}(t)=0$ then the equations of motion are sepa- 
rable: Hamilton's equations for $u$ and $v$ do not depend on one another. However, if a time-dependent field is present, the two degrees of freedom are coupled by a time-varying energy $\left(-p_{t}\right)$, and furthermore, the value of time along a trajectory depends on both $u$ and $v$. On the other hand, if initially $v(\tau=0)=0$ and $p_{v}(\tau=0)=0$, then they remain zero for all time, and the motion is completely described in the $\left(p_{u}, u, p_{t}, t\right)$ subspace.

\section{Approximate separation; quantization of action}

From the above remarks, it makes sense to make an approximate separation of variables by writing Eq. (62) as

$$
\begin{aligned}
& \frac{p_{u}^{2}}{2 \mu}+u^{2} p_{t}-2 e^{2}+\frac{e}{2} u^{4}\left[\mathcal{F}_{1}+\mathcal{F}_{2}(t)\right] \\
& \quad=-\left\{\frac{p_{v}^{2}}{2 \mu}+v^{2} p_{t}-\frac{e}{2} v^{4}\left[\mathcal{F}_{1}+\mathcal{F}_{2}(t)\right]\right\}=-\beta .
\end{aligned}
$$

This is exact before the pulse, with $\beta$ independent of time, and it is exact after the pulse, with $\beta$ again independent of time (though in general it could have a new value). Our assumption is that $\beta$ remains small enough that we can say it is nearly independent of time throughout the pulse. The validity of this approximate separation is explored in the Appendix.

Then the resulting approximate one-dimensional Hamiltonian for $l_{z}=0$ in atomic units is

$$
\tilde{H}_{1 \mathrm{D}}=\frac{1}{2} p_{u}^{2}+u^{2} p_{t}-2+\beta+\frac{1}{2} u^{4}\left[\mathcal{F}_{1}+\mathcal{F}_{2}(t)\right]=0 .
$$

By transforming back to cylindrical coordinates, Eq. (1) of Ref. [7] may be obtained. Note that after this transformation, $\beta$ becomes a small correction to the nuclear charge.

Before the pulse, we define action variables for $u$ and $v$ motion by using Eqs. (69) to express $p_{u}$ as a function of $u$ and $p_{v}$ as a function of $v$. (Both depend parametrically on $p_{t}=-E$ and on $\beta$.) Then

$$
\begin{aligned}
I_{u} & =\frac{1}{4 \pi} \oint p_{u}(u ; E, \beta) d u \\
I_{v} & =\frac{1}{4 \pi} \oint p_{v}(v ; E, \beta) d v .
\end{aligned}
$$

The semiclassical quantization for $I_{u}$ and $I_{v}$ are [16]

$$
\begin{gathered}
I_{u}=\left(n_{u}+1 / 2\right) \hbar \\
I_{v}=\left(n_{v}+1 / 2\right) \hbar .
\end{gathered}
$$

Note that the factors $1 / 4 \pi$ in front of the integrals [Eq. (71)] are unusual; they arise from the 2:1 mapping between $(u, v)$ and $(\rho, z)$.

The significance of $\beta$ can be seen again when we connect the quantization conditions to the energy spectrum. Before the pulse, suppose the static field is weak enough that

$$
\begin{aligned}
\left|\mathcal{F}_{1} v^{4} e / 2\right| & \ll\left|v^{2} p_{t}\right| \\
\left|\mathcal{F}_{1} u^{4} e / 2\right| & \ll\left|u^{2} p_{t}\right|
\end{aligned}
$$

Then Eqs. (69) are the equations of two separate harmomic oscillators, and the exact or semiclassical eigenvalues can be written down immediately:

$$
\begin{gathered}
\beta=2\left(n_{v}+1 / 2\right)\left(\frac{2 p_{t}}{\mu}\right)^{1 / 2} \hbar \\
2 Z e^{2}-\beta=2\left(n_{u}+1 / 2\right)\left(\frac{2 p_{t}}{\mu}\right)^{1 / 2} \hbar
\end{gathered}
$$

(with the extra factor of 2 in each equation due to our definition of action). Eliminating $\beta$ we obtain

$$
E=-\frac{\mu Z^{2} e^{4}}{2\left(n_{u}+n_{v}+1\right)^{2} \hbar^{2}} .
$$

On the other hand, if we had simply set $\beta$ to zero we would have obtained

$$
E=-\frac{\mu Z^{2} e^{4}}{2\left(n_{u}+1 / 2\right)^{2} \hbar^{2}} .
$$

Hence a nonzero value of $\beta$ is required to connect the correct half-integral quantization of the $u$ motion to the correct energy spectrum. [In past work it has been customary to set $\beta$ to zero, use the correct energy spectrum, and therefore use full-integer quantization of $I_{u}$. We propose that it is better to have $\beta$ nonzero (thus adjusting the effective nuclear charge) and use half-integral quantization of $I_{u}$. In practice, for large $n_{u}$ it does not make much difference.]

There is one final correction to the general theory outlined in Sec. II C for this particular system. The Maslov index changes when a trajectory (or path on a manifold) passes through $u=0$ [16], in addition to the specification given in Sec. II C. $p_{u}(u=0)$ can only take on two values: $\pm \sqrt{4-2 \beta}$. For the positive value, if $u$ goes from negative to positive $\mu$ is incremented, while if the sign of $u$ goes from + to,$- \mu$ is decremented. These rules are reversed for $p_{u}(u=0)=-\sqrt{4-2 \beta}$ and are valid whether they are evaluated in time along the classical trajectory or on the constant-time manifold.

\section{B. Numerical procedure}

A detailed description of how to obtain transition coefficients is given here. The requisite initial parameters are $n_{0}$, the initial quantum number; $\mathcal{F}_{1}$, the static field strength; $\mathcal{F}_{2}$, the peak amplitude of the time-dependent pulse; and $t_{p}$, the pulse length.

The equations of motion for $\left(p_{u}, u, p_{t}, t\right)$ are evaluated using Eqs. (63) $-(68)$ and setting $v=0$. However, because the $v^{2}$ term is dropped from Eq. (67), real time along the trajectory is not correct, the period of motion actualiy being too short [for zero electric field, $n_{v}=0, T_{1 \mathrm{D}}=$ 
$2 \pi n^{3}\left(1-\frac{1}{2 n}\right)$, which differs by $\pi n^{2}$ from the correct $2 \mathrm{D}$ result]. To compensate for this error, we scale time by $T_{2 \mathrm{D}} / T_{1 \mathrm{D}}$

Given $n_{u}=n-1$ and $n_{v}=0$, the energy of the initial state is solved for by numerical inversion of Eqs. (71a) and (71b) and using half-integer quantization of the action, as discussed in the preceding section. One then chooses a size $N$ for a grid in the initial angle variable coordinate with values between 0 and $2 \pi$. Each angle corresponds to a $\left(p_{u}, u\right)$ pair which can be found by starting at a turning point and successively integrating for time steps $T / N$. Thus one generates an initial manifold in phase space with position along it described by $\left(\Theta_{0}\right)_{k}=(k-1) 2 \pi / N T$ for integer $1 \leq k \leq N$.

Each initial condition is integrated in $\left(p_{u}, u, p_{t}, t\right)$ for the duration of the time-dependent pulse and then the action at the final energy is calculated using Eq. (71a) for each trajectory. For each of the allowed values of the action variable $\left(I_{n}=n_{u}+1 / 2\right)$ spanned by the values of action at the end of the pulse, the trajectories which lead to this final value of the action variable are analyzed. It is clear that $N$ must be chosen large enough that all intersections of $I\left(t_{f}\right)$ with $I_{n}$ are resolved. The exact initial conditions $\left(\Theta_{0}\right)$ for each of the trajectories with $I\left(t_{f}\right)=I_{n}$ are found by a root search. Then the slope $d I\left(t_{f}\right) / d \Theta_{0}$ [required in Eq. (18b)] is calculated by integrating a trajectory with a slightly different $\Theta_{0}$ (but the same $\left.I_{0}\right)$, and calculating $I\left(t_{f}\right)$ for that trajectory.

The only other information required for the transition probability is the action integral difference [the argument of the exponential in Eq. (18b)], which could in principle be found by evaluating an area on a plot of $\Theta\left(t=t_{f}\right)$ vs $I\left(t_{f}\right)$ (canonically conjugate variables). However, we found it most convenient to integrate the action integral along each trajectory in extended phase space $\left(p_{u}, u, p_{t}\right.$, $t)$ as well as that along the $\left(p_{u}, u\right) n$ manifold and express the relative phase as in Eq. (37b).

\section{ACKNOWLEDGMENTS}

This work was supported by the Office of Naval Research and the National Science Foundation. We thank JILA for their hospitality.

\section{APPENDIX: THE VALIDITY OF THE 1D APPROXIMATION}

Here we discuss some limitations of the onedimensional approximation, and we describe situations in which it seems likely that the $1 \mathrm{D}$ model will fail. We
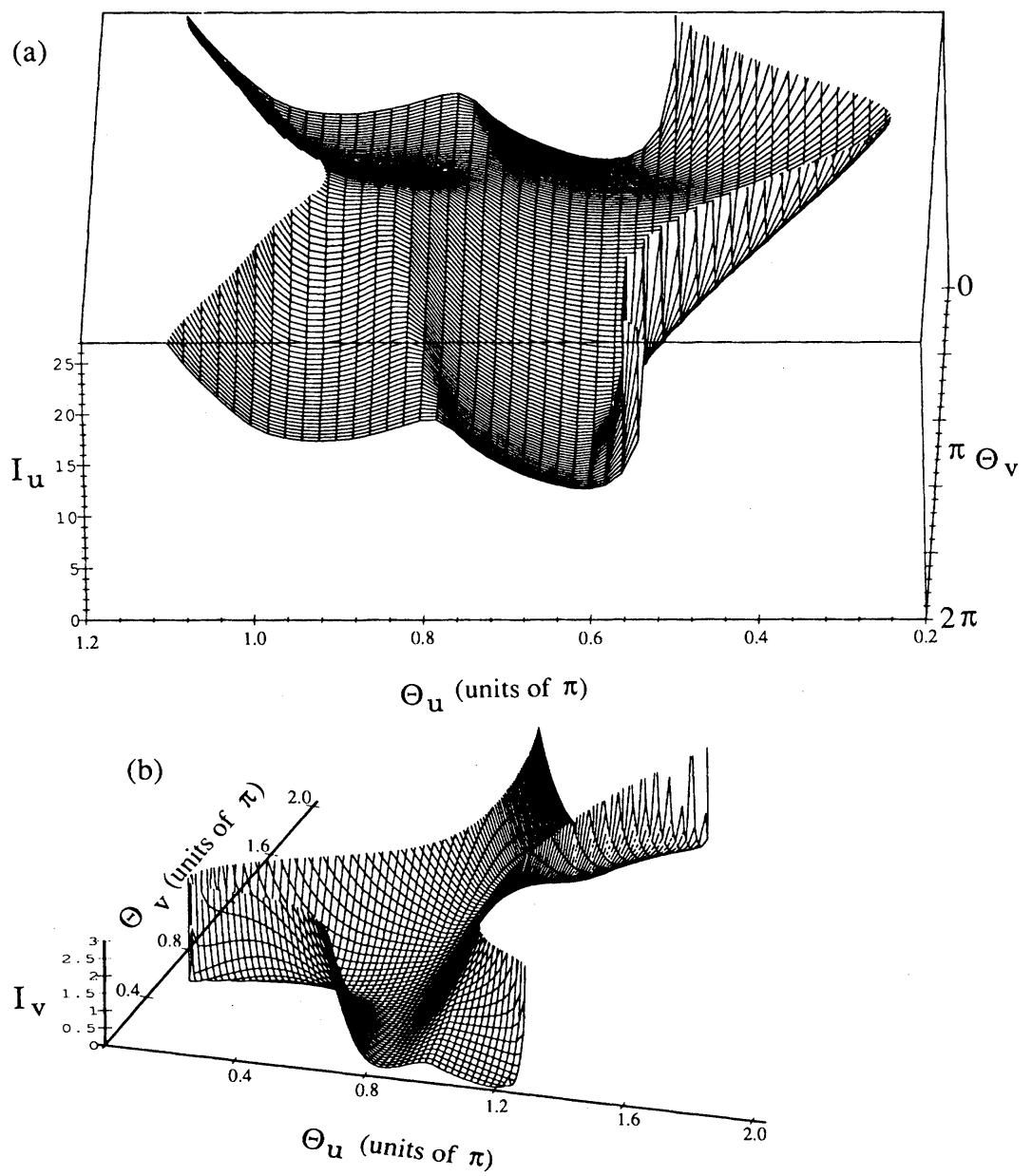

FIG. 2. Surface plots for (a) $I_{u}\left(t_{f}\right)$ and (b) $I_{v}\left(t_{f}\right)$ versus $\Theta_{u}(t=0)$ and $\Theta_{v}(t=0)$ after interaction with a half-cycle pulse for initial actions of $I_{u}=19.5, I_{v}=0.5$, and $\mathcal{F}_{2}=5 \times 10^{-6}$. 
compare the $1 \mathrm{D}$ approximation with a full $2 \mathrm{D}$ calculation for a case considered in the preceding paper.

From Eq. (69), the 1D model presumes that

$$
\frac{p_{v}^{2}}{2 \mu}+v^{2} p_{t}-\frac{e}{2} v^{4}\left[\mathcal{F}_{1}+\mathcal{F}_{2}(t)\right] \equiv \beta
$$

stays small, and close to its original value throughout the pulse. If $p_{t}(t)>0$ (negative energy) and $\mathcal{F} \equiv \mathcal{F}_{1}+\mathcal{F}_{2}<$ 0 , then the $v$ motion is confined by both terms in the effective potential energy. Whatever happens in the $u$ motion, we expect the $1 \mathrm{D}$ approximation to be accurate.

If $p_{t}(t)<0$ (positive energy) then the motion of the electron is unstable, and it will escape. If $\mathcal{F}<0$, it is the $u$ motion that is destabilized, while if $\mathcal{F}>0$ it is the $v$ motion that is destabilized. Either way the probability of ionization should be high; in the former case it should be described accurately by the 1D approximation, while in the latter case we expect the $1 \mathrm{D}$ approximation to fail.

Most interesting is the case $p_{t}>0$ (negative energy) and $\mathcal{F}>0$ (force on the electron toward the nucleus). Then the $u$ motion is confined, and the $1 \mathrm{D}$ approximation predicts no ionization. However, the effective potential for $v$ motion contains a barrier which becomes lower as $\mathcal{F}$ increases. An adiabatic approximation predicts that $v$ confinement will fail if the top of this barrier falls below $\beta(t)$.

This value of the field is found by using the equations for the action variables rewritten here as

$$
\begin{aligned}
I_{u} & =\frac{1}{4 \pi} \oint p_{u}(u ; E, \beta, \mathcal{F}) d u, \\
I_{v} & =\frac{1}{4 \pi} \oint p_{v}(v ; E, \beta, \mathcal{F}) d v .
\end{aligned}
$$

These action variables are the adiabatic invariants which remain constant under a slowly changing electric field. Changing $\mathcal{F}$ now results in changing $E$ and $\beta$, defined implicitly through these equations. However, for some value of $\mathcal{F}=\mathcal{F}^{*}$ the $v$ turning points will disappear, signifying destabilization of the $v$ motion. We find that, for $n_{v}=0$, the destabilization field strength of the blue states is (in a.u.)

$$
\mathcal{F}^{*}=4\left[\frac{2 \alpha}{\pi(n-1 / 2)}\right]^{4} \approx 0.3834\left(n-\frac{1}{2}\right)^{-4}
$$

where $\alpha=\int_{0}^{1} \sqrt{1-y^{4}} d y \approx 0.8740$. For comparison, the ionization field strength for the corresponding red states is $-1 /\left(16 n^{4}-8 n^{3}\right)$.

This failure is catastrophic in the adiabatic limit, and

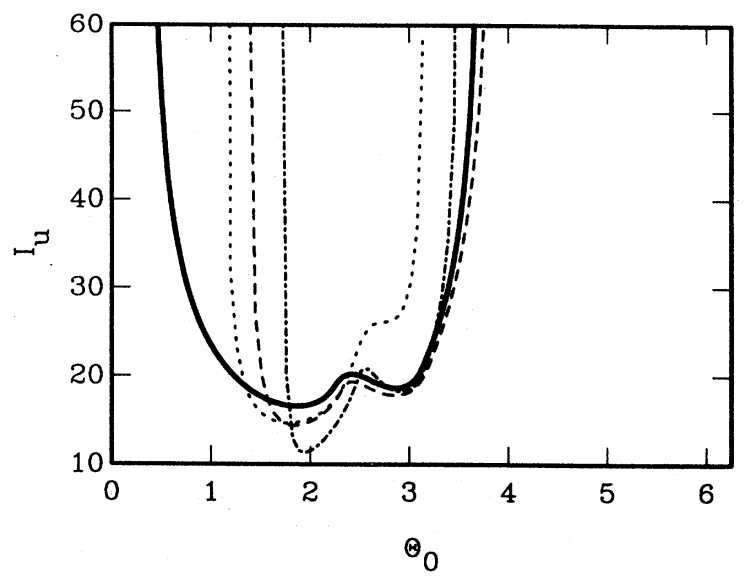

FIG. 3. Cross sections of the $I_{u}$ surface plot in Fig. 2(a) for $\Theta_{v}(t=0)=0.2 \pi$ (dashed curve), $\pi$ (dotted curve), and $1.7 \pi$ (dash-dotted curve). The $1 \mathrm{D}$ result is shown as the solid curve.

would lead to complete ionization and complete failure of the $1 \mathrm{D}$ approximation. Of course, if the field were to exceed this threshold for only a fraction of the Kepler period, then the $1 \mathrm{D}$ approximation may still give credible results.

Some of our calculations in the preceding paper involve fields that exceed this threshold; in particular the interesting bifurcation takes place in that regime. The pulse length was set to half the Kepler period, and the bifurcation involved an orbit that was close to the nucleus, and therefore catastrophic ionization and failure of the 1D approximation are not expected.

To examine this further, a 2D calculation was performed and the results compared to the predictions of the 1D model. Toward this end, an ensemble of trajectories with initial actions $I_{u}=\left(n_{u}+1 / 2\right) \hbar, I_{v}=1 / 2 \hbar$ and a distribution of conjugate angles $\Theta_{u}$ and $\Theta_{v}$ was integrated using Eqs. (63)-(68). After interacting with the pulse, each trajectory was analyzed for $I_{u}\left(t_{f}\right)$ and $I_{v}\left(t_{f}\right)$. Surface plots for $I_{u}\left(\Theta_{u}(0), \Theta_{v}(0)\right)$ and $I_{v}\left(\Theta_{u}(0), \Theta_{v}(0)\right)$ are shown in Figs. 2(a) and 2(b), respectively, for $n_{u}=19$, $n_{v}=0, \mathcal{F}_{1}=0, \mathcal{F}_{2}=5 \times 10^{-6} \approx 2 \mathcal{F}^{*}$. Cuts through one of these surfaces are shown in Fig. 3. We see that (1) many orbits survive with finite values of $I_{u}$ and small values of $I_{v} ;(2)$ the bifurcation persists in the $2 \mathrm{D}$ calculation. It is manifested in the double-minimum structure of $I_{u}\left(\Theta_{u}, \Theta_{v}\right)$. We conclude that even in this case the 1D approximation is at least qualitatively correct.
[1] P. A. M. Dirac, Proc. R. Soc. London, Ser. A 110, 561 (1926); 111, 281 (1926); 114, 243 (1927).

[2] A good history may be found in J. Mehra and $H$. Rechenberg, Historical Development of Quantum Theory (Springer-Verlag, New York, 1982), Vol. 2.

[3] P. Carruthers and M. M. Nieto, Rev. Mod. Phys. 40, 411 (1968).

[4] W. H. Miller, Adv. Chem. Phys. 25, 69 (1974).

[5] R. A. Marcus, Chem. Phys. Lett. 7, 525 (1970); J. Chem. Phys. 54, 3965 (1971).
[6] V. P. Maslov and M. V. Fedoriuk, Semi-Classical Approximation in Quantum Mechanics (Reidel, Dordrecht, 1981).

[7] C. D. Schwieters and J. B. Delos, preceding paper, Phys. Rev. A 51, 1023 (1995).

[8] J. B. Delos, R. L. Waterland, and M. L. Du, Phys. Rev. A 37, 1185 (1988).

[9] J. E. Bayfield and L. A. Pinnaduwage, Phys. Rev. Lett. 54, 313 (1985). 
[10] R. V. Jensen, Phys. Rev. A 30, 386 (1984).

[11] C. Chester, B. Friedman, and F. Ursell, Proc. Cambridge Philos. Soc. Math. Phys. Sci. 53, 599 (1957).

[12] F. Ursell, Proc. Cambridge Philos. Soc. 72, 49 (1972).

[13] J. N. L. Connor, Mol. Phys. 26, 1217 (1973).
[14] T. Pearcey, Philos. Mag. 37, 311 (1946).

[15] V. I. Arnold, Mathematical Methods of Classical Mechanics, 2nd ed. (Springer-Verlag, New York, 1989).

[16] J. Gao and J. B. Delos, Phys. Rev. A 46, 1455 (1992). 